Área Abierta. Revista de comunicación

audiovisual y publicitaria

ISSN: 2530-7592

\title{
Serial, el Programa Radiofónico que Resucitó el Podcasting
}

\author{
Rafel Linares de Palomar'; Elena Neira Borrajo ${ }^{2}$
}

Recibido: 2 de septiembre de 2016 / Aceptado: 14 de diciembre de 2016

Resumen. El podcast Serial comienza su emisión en octubre de 2014, lo que supone un cambio radical en el modelo de este nuevo formato que se populariza y consigue atraer la atención de la inversión publicitaria. Desde este momento, el aumento de la inversión económica, la proliferación de aglutinadores de estos formatos, la profesionalización y el interés de los grandes grupos de comunicación hacen renacer el podcasts como medio de comunicación. En el siguiente artículo proponemos un estudio de caso de Serial como fenómeno que revolucionó el podcasting.

Palabras clave: Podcast, Serial, podcasting, nuevos modelos de negocio, series, radiodifusión

\section{[en] Serial, The Radio Programme that Brought Podcasting Back to Life}

\begin{abstract}
The broadcast of Serial, a podcast released in October 2014, triggered a radical change in the model of this new format, making it more popular for audiences and attractive to advertising incomes. Since that moment, the increase of investment, aggregators and the big media groups' interest revive podcasting as a communication tool. This paper will focus on Serial as a study case. Keywords: Podcast, Serial, Podcasting, New Businesss Model, Series, Radio Broadcasting
\end{abstract}

Sumario. Introducción. 1. Estado de la cuestión. 2. Objetivos y metodología. 3. Serial, el podcast que atrajo la atención al medio. 4. Discusión y conclusiones. 5. Bibliografía.

Cómo citar: Linares de Palomar, R. y Neira Borrajo, E. (2017) Serial, El Programa Radiofónico Que Resucitó El Podcasting, en Área Abierta. Revista de comunicación audiovisual y publicitaria 17 (1), 73-82. http://dx.doi.org/10.5209/ARAB.53356

$1 \quad$ Universidad Rey Juan Carlos rafael.linares.palomar@urjc.es

2 Universitat Oberta de Catalunya eneira@uoc.edu 


\section{Introducción}

La estabilidad del modelo radiofónico en España basado en el oligopolio de la época de las transmisiones hercianas poco se vio alterada con la introducción de nuevas tecnologías como el Digital Audio Broadcasting (DAB), la TDT o Internet. El fracaso de la radio digital DAB en España, independientemente de los problemas económicos e industriales, vino causado por la búsqueda de las radios de un nuevo soporte de difusión en Internet. Al principio, la web fue un espacio para volcar la información de las estrellas de las radios nacionales, comenzando las primeras emisiones en el año 1995, aunque el concepto de radio a la carta tiene que esperar hasta septiembre de 1996 con Catalunya Radio siendo la cadena que lo inaugura (Franquet, 2002: 210).

Realmente, no es hasta el año 2001 con la aparición del iPod, cuando comienza a surgir una ruptura desde dos puntos de vista, el narrativo y el empresarial. Por un lado, proliferan creaciones de audio digitales, ajenas a los grupos multimedia habituales y a las grandes figuras del periodismo radiofónico tradicional. Por otro lado, estas narrativas están realizadas por personas ajenas a la tradicional industria de la radio. El fácil acceso a medios técnicos y la sencillez del manejo de los mismos comienza a generar la proliferación de nuevos creadores que innovan tanto en contenidos como en nuevos modelos de negocio, todavía marginales y muy lejos de la rentabilidad económica que exige una industria tradicional.

Aunque aún quedarían algunos años para la creación de Youtube (2005) y la proliferación de los videobloggers, ahora convertidos en youtubers, con la aparición del podcast comienza a generarse la figura del prosumer radiofónico tal y como la define Scolari. Son creadores que «van más allá del consumo tradicional y se convierten en productores de nuevos contenidos (prosumidores) bajo las banderas del remix y la postproducción» (2013: 222).

Ya corresponda el uso de los podcast a creaciones radiofónicas realizadas por individuos o a acciones de estrategias de programación de los medios de comunicación para expandir su programación en diferentes ventanas y medios, entran dentro de la lógica de las narrativas transmedia, como las define Jenkins: «una historia transmediática se desarrolla a través de múltiples plataformas mediáticas y cada nuevo texto hace una contribución específica y valiosa para la comunidad» (2008: 101).

Los podcast quedan configurados como una publicación periódica digital de audio que se descarga de Internet a través de una sindicación web, por lo que «se cambia de esta forma, no solo la manera tradicional de elaborar, estructurar y enviar el mensaje, sino las nuevas formas de consumo e interacción con los oyentes o usuarios de las webs radiofónicas» (Chomón Serna, 2016: 263). Esta facilidad y accesibilidad ha permitido, como indica Ortiz Sobrino (2010), la proliferación del periodismo ciudadano o colaborativo, generando nuevos contenidos que obligan a las tradicionales estructuras radiofónicas a adaptarse para la creación de nuevos recursos y acciones que ayuden a tener una mayor capacidad de atracción en la audiencia.

El surgimiento de estos contenidos encapsulados encaja con la lógica de la nueva economía de The Long Tail (Anderson, 2007), basada en la producción de contenidos con costes mínimos y orientados a mercados masivos, divididos en millones de nichos, gracias a las nuevas características que ofrece la distribución online (gastos 
mínimos de almacenaje y distribución ilimitada). Por ello, «la Red permite estimular la creación y venta de productos dirigidos a públicos minoritarios que, considerados de forma agregada, constituyen una demanda importante» (Izquierdo-Castillo, 2012: 386). Asociada a esta línea, y como bien identifican Merayo y Pedrero (2001), Internet permite en los productos radiofónicos la hipersegmentación, el aumento de las horas de producción, el salto internacional a países con lengua común y la generación de valores añadidos.

\section{Estado de la cuestión}

Pero la realidad en estos primeros años en torno a la industria del podcast es muy frustrante. Apenas existe un interés por parte de la publicidad para destinar parte de su presupuesto en medios a este formato, lo que hace que se mantenga durante una década un ecosistema ajeno a la economía de mercado y con una infraestructura ínfima. Así, ha quedado relegado a un canal de distribución menor para las radios, donde simplemente replican su programación radiofónica, o para creadores amateurs sin grandes aspiraciones en retornos económicos. Hay que esperar hasta 2013, refiriéndonos a Estados Unidos, para que surja un incremento del interés por estos productos sobre todo asociados al fenómeno de la conectividad en los coches y la penetración móvil. La mayor atención a este producto viene dentro de la ola de los nuevos usos asociados a los medios tradicionales, como la movilidad en la música, con Spotify o Deezer, o la televisión en plataformas como Netflix o Hulu. Además los podcast mantienen ciertas ventajas que los hacen muy atractivos: escasa inversión para su producción, publicidad nativa directa y estratificada y el abaratamiento y mejoras al acceso de datos desde la telefonía móvil (4G).

Es en el último trimestre de 2014 cuando se estrena, Serial, el podcast radiofónico objeto de nuestro análisis y que supone un punto de inflexión. Su alto número de descargas y su influencia social ha supuesto que algunos medios (Fast Company, The Guardian) identifiquen el año 2014 como el año del Podcast Renaissance, el renacimiento del podcast. Como es habitual, es necesario crear un fenómeno de masas para la introducción y popularización de una nueva tecnología, a modo de estrategia de punta de lanza. Frente a la economía de The Long Tail, las estrategias empresariales de la industria del entretenimiento obtienen más ingresos cuando invierten en productos superventas (Elberse, 2014 y 2008) y Serial es un claro ejemplo de estas acciones, aunque con presupuestos bajos comparados con otras producciones audiovisuales. Estos nuevos formatos de contenido están «alejados de la lógica empresarial que todavía hoy reproducen las estructuras organizativas mastodónticas de la radio pública, y a diferencia de criterios organizativos llevados a cabo por las grandes cadenas comerciales, estos modelos de gestión low cost se caracterizan por la máxima optimización de sus recursos» (Ortiz Sobrino, 2012).

El incremento de la popularidad a raíz de esta serie y acciones como la introducción de una app obligatoria de podcast en el iOS 8 de Apple -en junio de 2014- supuso un aumento en la inversión publicitaria, donde las marcas veían un medio directo con un gran potencial en el que anunciar sus productos a una audiencia enganchada con altos grados de empatía, gran carga emocional y con un alto interés de atención e intimidad. Aunque la situación en España no ha sido tan inmediata. El grupo Prisa 
se ha incorporado a esta tendencia, a través de la creación de Podium Podcast -un agregador de podcast cuyo objetivo es convertirse en la red global en castellano más importante del mundo-, aprovechando el poco desarrollo del sector y la escasa competencia (tan solo Invoox y Cuonda, plataformas especializadas en contenidos en castellano). Curiosamente, justo un mes antes, en la páginas de economía de El País, publicaban una extensa noticia sobre cómo «del podcast no vive nadie» (Abad, 2016).

A pesar del interés despertado por los podcast, existe una serie de retos a los que deberá enfrentarse para su consolidación. En primer lugar, tiene que hacer frente a una fuerte atomización de webs con contenidos a la par que a una profunda concentración en torno a agregadores (Apple o Ivoox). Otro problema es la indexación, dado que aún no está vinculado el audio a Google ni a otros buscadores. Y, por último, no existe una estandarización de la industria en las métricas, ni acuerdos con entidades que regulen las normas, incluso en los datos que se ofrecen se habla de descargas y no de escuchas reales.

\section{Objetivos y metodología}

El objetivo general de la presente investigación es analizar el caso que revolucionó la incipiente industria del podcast, pasando de ser un mercado minoritario y despreciado por los inversores publicitarios a convertirse en un medio altamente atractivo como canal de comunicación debido a las ventajas que ofrece. En concreto, el objetivo es el estudio del podcast como herramienta de producción de ficción y no ficción, diferenciada de otras fórmulas de producción tradicionales. Internet ha abierto las puertas a nuevos formatos de ficción, tanto en duración como en distribución y los podcast son un claro ejemplo de la ruptura con los medios de antaño.

Este trabajo se basa en un estudio de caso, la primera temporada del podcast Serial, que presenta como hecho significativo ser el primer gran éxito de la industria, mezclando una narrativa ficcionada sobre un caso real. El análisis de la investigación sobre el que se desarrolló, su forma de emisión, su campaña de marketing y la expansión del formato mediante contenido puente web, permiten categorizar los elementos que le ha llevado al éxito, tanto sociales, como promocionales y narrativos. El estudio es de una naturaleza no experimental y con un eminente carácter descriptivo, ya que su principal objetivo es definir el fenómeno y su transcendencia.

El desarrollo de la investigación se estructura en tres etapas: exploratoria, analítica y conclusiva, en un marco temporal marcado por el inicio de la emisión, el 3 de octubre de 2014 hasta su conclusión, tres meses más tarde, lo que nos permite el análisis de Serial como caso de estudio completo. Para la investigación se ha hecho uso de fuentes externas para recopilar la información que nos permita plantearnos las hipótesis que intentamos responder.

¿Es Serial el primer podcast que ha supuesto un cambio en esta industria? ¿Cómo consigue el programa mantener fidelizada a la audiencia? ¿Es necesaria la introducción de elementos transmedia en una narrativa de podcast? Los autores son conscientes de la limitación de extrapolar el caso de estudio a la industria, pero consideran Serial como un elemento clave que ha articulado un cambio en el podcasting internacional. 


\section{Serial, el podcast que atrajo la atención al medio}

En octubre de 2014 se estrenaba el podcast Serial. Nacía como un spin off de This American Life, un programa radiofónico, de corte periodístico, emitido en más de 500 canales de radio y con una audiencia media de más de 2 millones de oyentes ${ }^{3}$. A las pocas semanas de su lanzamiento ya se hablaba de fenómeno. Como indica Berry (2015a), el patrón de consumo de Serial empezó «con los early adopters que descubren algo nuevo para luego compartir esa experiencia con otros mediante su recomendación personal». Esta situación acabó desencadenando una histeria colectiva entre la población norteamericana. Casi 40 millones de estadounidenses declararon haber escuchado algún episodio de Serial, según una encuesta de Edison Research (2014).

Y no tardó en contagiarse al resto del mundo. Serial, «una historia contada semana a semana» (según reza la entradilla del programa), se convirtió en el centro de comentarios de decenas de foros, en el tema estrella de hilos de conversación en Reddit, en motor de contenido generado por el usuario en Twitter y Facebook, en protagonista de seminarios de investigación amateur o en caso de estudio en varios institutos. La prensa especializada llegó, incluso, a equipararlo con el impacto que tuvo la emisión en televisión de la célebre Twin Peaks (Ayuso, 2014). Fue, en definitiva, el primer podcast que logró convertir en mainstream un formato hasta entonces minoritario.

La primera temporada de Serial se articuló en entregas semanales de entre 40 y 50 minutos de duración que los usuarios podían escuchar en streaming o descargar de Internet. Cada nuevo episodio se publicaba los jueves por la mañana (solo el puente de Acción de Gracias rompió la dinámica).

La serialización tradicional, con sus mecanismos narrativos básicos como gancho para el oyente, se combinó con un marketing de programa que fomentaba en cada emisión la suscripción (tanto al podcast a través de las apps móviles como al newsletter web). Igualmente, se animaba a la audiencia a estar puntualmente informada de cualquier información o novedad.

Las cifras del debut de Serial supusieron un contagio a otros podcast (Roberts, 2014). El interés popular catapultó la docuserie al número uno de descargas de iTunes durante más de tres meses (Estados Unidos, Canadá, Reino Unido y Australia) y a ser escogido como el mejor podcast estrenado en ese año por parte de dicha plataforma (Whitney, 2014). Con un promedio de audiencia de 1,5 millones de oyentes por episodio (con picos de hasta 2,2 millones) fue el primer podcast que más rápido superó los cinco millones de descargas (Dredge, 2014). Como indica Berry (2015a) «Serial se convirtió en un tema muy debatido en los entornos sociales, en los medios tradicionales, y de manera significativa en una gran cantidad de podcasts sobre el podcast».

Por parte de la crítica, todo fueron elogios: «el primeréxito reciente del podcasting» (Carr, 2014), «un nuevo género de storytelling de audio» (Sawyer, 2014), «un fenómeno inesperado» (Gordon y Bond, 2014), «un podcast cautivador» (Shultz, 2014). La fórmula se basaba en elaborar una historia que atrapaba y una manera innovadora de relatarla. Ira Glass, showrunner del programa, declaraba poco antes

3 Datos oficiales de la web del programa. Recuperado de http://www.thisamericanlife.org (Fecha de acceso 30/06/2016). 
del lanzamiento del primer episodio, que su intención era proporcionar la misma experiencia que ofrecía una serie de HBO o Netflix. Al igual que dichos productos seriados, el podcast recurría al descubrimiento semanal de tramas y personajes, y apostaba por el establecimiento de una relación mantenida con un universo narrativo. De esta manera alimentaban el ansia por saber qué ocurriría a continuación. Glass, lo compara con Juego de Tronos y añade que Serial puede entretener mientras se conduce (2014). Serial aportaba dos elementos diferenciales. Primero, la historia era real -no estando, en sentido estricto, dramatizada-, y, segundo, dada su naturaleza radiofónica, carecía de imágenes durante la emisión -aunque se contrarrestaba con un potente universo gráfico digital que se actualizaba semanalmente en su página web-.

El equipo de Serial se propuso el ambicioso objetivo de reabrir la investigación de un crimen ocurrido hacía quince años: el asesinato de una adolescente de origen surcoreano (Hae Min Lee) en Maryland (Baltimore, EE.UU.). El caso llegó a manos de la productora a través de Rabia Chaubry, amiga de la familia del condenado. Chaubry llevaba varios años denunciando en los medios las irregularidades del juicio por el asesinato de Hae Min Lee para conseguir reabrir la investigación. Adnan Syed, el presunto asesino convicto y exnovio de la víctima, se encuentra cumpliendo cadena perpetua. El imaginario colectivo recreado a través de fuentes literarias o televisivas toma forma en esta investigación: adolescentes queridos por sus amigos, una coartada frágil, una investigación policial apresurada, una llamativa ausencia de pruebas materiales en el escenario del crimen, así como una defensa judicial plagada de reproches.

Es indudable que tras Serial había una historia con potencial para llamar la atención, pero sorprende que un producto tan tradicional pudiera atraer la atención de millones de personas. Gran parte del mérito es de su conductora, la periodista Sarah Koenig, cuyo estilo narrativo ha sido comparado con el de Truman Capote o Charles Dickens (González, 2014). Con un tono cercano e informal, y una dicción impecable, Koenig fue el vehículo principal en un programa en el que la reconstrucción pretendía ser completa para que el oyente tuviese todas las claves necesarias. En cada episodio eran frecuentes las entrevistas a las personas involucradas, las conversaciones telefónicas con el propio Adnan Syed desde el teléfono público de la prisión a cobro revertido, los interrogatorios llevados a cabo en las dependencias de la policía, el audio con las declaraciones de los abogados y testigos en el juicio. Serial propuso un híbrido entre la crónica periodística y el entretenimiento radiofónico. La fuerza de su debut radicó, precisamente, en tratar sobre un hecho real dramático, pero narrativamente imperfecto, de ahí la ausencia de recursos para conseguir la suspensión de incredulidad, en la denominación acuñada por Samuel Taylor Coleridge. El formato seriado permitía profundizar lo suficiente para abordar cada elemento del caso con detenimiento y ofrecer diferentes enfoques, pruebas, entrevistas, reconstrucciones, testimonios y pensamientos a modo de palanca de fidelización, es decir, generando la necesidad de saber más de la historia.

El trabajo de Koenig consistía en informar, no en exonerar (Fresh Air, 2014). Sin embargo, no pudo evitar que sus propias dudas y pensamientos afloraran con cada hallazgo, cada nuevo punto de vista, testigo y experto entrevistado. Serial se convirtió, además de en crónica, en un acto íntimo. La complicidad que alimentó la presentadora animaba al oyente a zambullirse en una historia cuajada de interrogantes y contradicciones, que no se podía dejar a medias. Algo a lo que también contribuyó 
una cuidada atmósfera auditiva, pensada para ser escuchada en soledad y con total atención.

Uno de los principales retos que afrontaba Serial era el de conquistar a una audiencia educada en historias más o menos estructuradas, con dos o tres giros inesperados y un final conclusivo. Pero Serial se basaba en una historia real. Y a diferencia de la ficción, las historias reales no tienen por qué tener sentido. La premisa ya asumía un reto: burlar la necesidad de una resolución cognitiva por parte de la audiencia (Singal, 2014). Por ello se usó la incertidumbre como un elemento más de la propia narración. Algo que también terminó influyendo en la propia producción del programa. Sarah Koenig no ocupaba la posición de un narrador omnisciente. Los hechos se presentaban uno detrás de otro. Y la audiencia se formaba una opinión casi en paralelo. Se trabajaba sin el guion del episodio siguiente y sin tener muy claro cómo iba a terminar la historia. Su potencial adictivo, en definitiva, fue fruto de la convergencia entre una buena historia y su peculiar modelo narrativo. Dos elementos que, combinados, fueron capaces de movilizar a la comunidad y traspasar las fronteras territoriales y culturales. Circunstancia que no se produjo en la segunda temporada, debido a la ausencia de empatía con el protagonista, el soldado americano Bowe Bergdahl que desertó de su base y pasó cinco años retenido por los talibanes.

Serial, además, superó las limitaciones del formato podcast planteando una auténtica ficción interactiva y transmedia. Los hechos reales colocaban al podcast ante el reto de generar un buen contenido puente entre emisiones para alimentar el interés mientras se esperaba a la siguiente entrega: planteando los interrogantes del caso, reconstruyendo líneas temporales, desgranando las declaraciones de los testigos, entre otros. Como apunta Richard Berry (2015a), «Serial lo que hizo fue ofrecer un podcast que no solo tenía atractivo de masas, sino que también se presentaba como una narrativa en la que el público podía involucrarse intelectual y emocionalmente». La semana entre emisiones se acabó convirtiendo en un período de reflexión, lo que permitía que los seguidores interactuasen. La web de Serial actuó como versión 2.0 de Elige tu propia aventura en la que cada uno decidía qué camino de la investigación recorrer: el escrutinio de los interrogatorios, la lista del móvil de Adnan, las declaraciones de expertos, croquis de testigos, fotografías de las personas involucradas, infografías, reconstrucciones, cartas, extractos del diario de la víctima. Apelando a la curiosidad, aportando datos y desafiando a darles sentido. Muchos oyentes se convirtieron en improvisados investigadores del caso. Los hechos reales se vieron, de esta manera, propulsados por una narración verdaderamente social, alimentada por un motor colaborativo. Y el éxito de audiencia multiplicó los efectos. A mayor popularidad del podcast, mayores recursos. Más oídos. Más datos. Más testimonios. Más visiones. Como ejemplo, observamos el episodio 9 que comienza con tres revelaciones de la audiencia surgidas a raíz de la escucha del podcast. Este caso indica la importancia del contenido generado por el equipo de producción del programa y la aportación de la audiencia -fenómeno fan-. Sobre estas contribuciones, surgieron productos tan sorprendentes como el podcast sobre el podcast (Serial Spoiler Special de Slate), que dedicaba semanalmente su programa a hacer un repaso de la emisión). Además, se puede encontrar un elevado número de teorías de la conspiración generadas por usuarios en Reddit.

Gracias a la transcendencia mediática e interés de la audiencia, Serial, ha sido clave en el proceso judicial. El pasado 5 de julio de 2016, Sarah Koenig informaba a los fans de Serial a través del blog del programa que el juez Martin P. Welch había 
dictaminado que Syed tendría derecho a un nuevo juicio. La decisión se basó en que su primera abogada ya fallecida, Cristina Gutiérrez, no había contactado con una testigo clave, quien habría proporcionado una coartada al acusado. Se trata de Asia McClain, entrevistada para Serial, y que aseguró haber visto a Adnan a la misma hora en la que Hae fue asesinada. En dicha entrada se pueden escuchar los audios de las conversaciones que Koenig ha tenido con Adnan, en las que los oyentes pueden escuchar, de primera mano, cómo ha recibido la noticia y cuáles son sus expectativas ante esta nueva oportunidad para defender su inocencia.

Hollywood ya ha declarado su interés por la historia (Abramovitch, 2014) y la $\mathrm{BBC}$ ha adquirido los derechos para adaptar la serie a la televisión, documentando el proceso de producción del programa de radio. Aunque la acogida de la segunda temporada ha sido más tibia de lo esperado (Locker, 2016), ya nadie duda de que Serial ha acuñado una marca rentable y reputada que ha contribuido, de manera decisiva, a relanzar el interés popular de la radio.

\section{Discusión y conclusiones}

Es difícil extraer conclusiones dada la peculiaridad del caso y la limitación del modelo de trabajo propuesto, pero aun teniendo presentes estas cautelas y sopesando trabajos publicados como los de Richard Berry (2015a y 2015b) podemos responder a algunas de las preguntas que nos hacíamos en nuestras hipótesis. Por un lado, entendemos que Serial ha dado un giro importante en el desarrollo de la industria del podcast, otorgándole una relevancia que hasta ese momento no tenía.

Las motivaciones que han llevado a este cambio pueden ser varias, como hemos ido viendo a lo largo del estudio. Una audiencia altamente enganchada y con un gran nivel de atención ante un caso que, aunque esté ficcionado parte de un hecho real. La alta generación de recursos multiplataformas que se han generado en torno a Serial, especialmente la propia web del programa, ofreciendo muchos recursos a los seguidores. A ello se ha sumado la alta participación de la audiencia, siguiendo el modelo de consumidor que crea (prosumer), propuesto por Jenkins, ya sea con su participación en redes sociales, o en la generación de otros podcast que han creado un universo paralelo al original.

También consideramos que es importante destacar cómo Serial es coetánea a una de las series con mayor impacto en audiencia y relevancia en la producción audiovisual, como es True detective (2014), que se acerca también desde la ficción a la resolución de un crimen sin resolver. Ello nos hace pensar y, queremos destacar, cómo resurge en años posteriores del denominado crimen documental, con la ola de relatos reales ficcionados como American Crime Story, con su primera temporada The People v. O.J. Simpson (FOX, 2016), la serie Making a Murderer (Netflix, 2015), o la miniserie La mala suerte: La vida y la muerte de Robert Durst (HBO, 2015), todas teniendo como elemento común la ficción de un asesinato real en formato documental. 


\section{Bibliografía}

Abad, J.M. (2016). «Del podcast no vive nadie». El País. Recuperado de http://economia. elpais.com/economia/2016/05/06/actualidad/1462529204_717336.html (Fecha de acceso 31/07/2016).

Abramovitch, S. (2014). «Of Course Hollywood Producers Are Now Wooing 'Serial'». The Hollywood Reporter. Recuperado de http://www.hollywoodreporter.com/news/coursehollywood-producers-are-wooing-750011 (Fecha de acceso: 30/06/2016).

Anderson, C. (2007). La economía Long Tail. Barcelona: Urano.

Ayuso, S. (2014) «'Serial', la serie de radio que tiene en vilo a millones de estadounidenses».

El País. Recuperado de http://internacional.elpais.com/internacional/2014/12/08/ actualidad/1418059208_072805.html (Fecha de acceso: 30/06/2016).

Berry, R. (2015a). «A Golden Age of Podcasting? Evaluating Serial in the Context of Podcast Histories». Journal of Radio \& Audio Media 22, 2, pp. 170-178. DOI: 10.1080/19376529.2015.1083363.

- (2015b). «Serial and Ten Years of Podcasting: Has The Medium Finally Grown Up?». En M. Oliveira y F. Ribeiro (Eds.), Radio, Sound and Internet. Braga: LASICS, pp. 299-309.

Carr, D. (2014). "'Serial', Podcasting's First Breakout Hit, Sets Stage for More». The New York Times. Recuperado de http://www.nytimes.com/2014/11/24/business/media/ serial-podcastings-first-breakout-hit-sets-stage-for-more.html?_r=0 (Fecha de acceso: 30/06/2016).

Chomón Serna, J.M. (2016): «La radio glocal cross-media: la alternativa ante la reducción de espacios de proximidad local en la radio convencional». Icono 14, 14, 1, pp. 258-286. DOI: $10.7195 /$ ri14.v14i1.894.

Dredge, S. (2014). «Serial Podcast Breaks iTunes Records as it Passes 5m Downloads and Streams». The Guardian. Recuperado de https://www.theguardian.com/technology/2014/ nov/18/serial-podcast-itunes-apple-downloads-streams. (Fecha de acceso: 30/11/2016).

Edison Research. (2014). «The Infinite Dial». Recuperado de http://www.edisonresearch. com/wp-content/uploads/2014/03/The-Infinite-Dial-2014-from-Edison-Research-andTriton-Digital.pdf (Fecha de acceso: 30/06/2016).

Elberse, A. (2008). «Should You Invest in the Long Tail?». HBS Centennial Issue Harvard Business Review, 86, 7/8, pp. 88-96.

- (2014). Superventas. Barcelona: Gestión 2000.

Franquet, R. (2002): «La radio en el umbral digital: concentración versus diversificación». En E. Bustamante (Ed.), Comunicación y cultura en la era digital. Industrias, mercados $y$ diversidad de España. Barcelona: Gedisa, pp. 179-212.

Fresh Air. (2014). «Serial Host Sarah Koenig Says She Set Out To Report, Not Exonerat». Recuperado de http://www.npr.org/2014/12/23/372577482/serial-host-sarah-koenigsays-she-set-out-to-report-not-exonerate (Fecha de acceso 30/06/2016).

Glass, I. (2014). «Announcing Serial». This American Life Blog. Recuperado de http://www. thisamericanlife.org/blog/2014/07/announcing-serial (Fecha de acceso: 30/06/2016).

González, J. (2014). "'Serial', el adictivo podcast que tiene a millones siguiendo un asesinato». $B B C$. Recuperado de http://www.bbc.com/mundo/noticias/2014/11/141112 eeuu_podcast_serial_exito_crimen_jg (Fecha de acceso: 30/06/2016).

Gordon, S. y Bond, S. (2014). «'Serial': Inside a Podcast Phenomenon». Financial Times. Recuperado de http://www.ft.com/cms/s/2/aeb8d37c-7af1-11e4-8646-00144feabdc0. html (Fecha de acceso: 30/06/2016).

Izquierdo-Castillo, J. (2012). «Distribución online de contenidos audiovisuales: análisis de 
tres modelos de negocio». El profesional de la información, 21, 4, pp. 385-390. DOI: http://dx.doi.org/10.3145/epi.2012.jul.09.

Jenkins, H. (2008). Convergence Culture: La cultura de la convergencia de los medios de comunicación. Barcelona: Paidós Comunicación.

- (2009). Fans, blogueros y videojuegos. La cultura de la colaboración. Barcelona: Paidós Comunicación.

Locker, M. (2016). «Serial Season Two: Why did the 'Must-listen Show' Suffer a Sophomore Slump». The Guardian. Recuperado de https:/www.theguardian.com/tv-and-radio/2016/ apr/05/serial-season-two-bowe-bergdahl-podcast (Fecha de acceso: 30/06/2016).

Merayo, A. y Pedrero, L. M. (2001). «Hacia un modelo radiofónico de convivencia y convergencia». En Informe anual de la comunicación 2000/01. Madrid: Zeta, pp. 337342.

Ortiz Sobrino, M. A. (2012). «Radio y post-radio en España: una cohabitación necesaria y posible». Área Abierta, 12, 2, pp- 1-16. DOI: http://dx.doi.org/10.5209/rev_ARAB.2012. n32.39637

Roberts, A. (2014). «The Serial Podcast: by the Numbers». CNN . Recuperado de http:// edition.cnn.com/2014/12/18/showbiz/feat-serial-podcast-btn/ (Fecha de acceso: 30/06/2016).

Schultz, A. (2014). «Review: Serial, a Captivating New Podcast». The Daily Californian. Recuperado de http://www.dailycal.org/2014/10/10/serial-podcast/ (Fecha de acceso: 30/06/2016).

Scolari, C. A. (2013). Narrativas Transmedia. Cuando todos los medios cuentan. Barcelona: Deusto.

Singal, J. (2014). «A Psychological Explanation for Why 'Serial' Drives Some People Crazy». Science of Us. Recuperado de http://nymag.com/scienceofus/2014/12/why-serial-drivessome-people-crazy.html (Fecha de acceso: 30/06/2016).

Swayer, M. (2014). «Serial Review: the Greatest Murder Mystery you Will Ever Hear». The Guardian. Recuperado de https:/www.theguardian.com/media/2014/nov/08/serialreview-greatest-murder-mystery-ever-hear (Fecha de acceso: 30/06/2016).

Whitney, L. (2014). «Serial Podcast, Lego Movie, Beyonce Rule Best of iTunes 2014». CNET. Recuperado de https://www.cnet.com/news/apple-reveals-its-best-of-itunespicks-for-2014/ (Fecha de acceso: 30/11/2016). 